\title{
Sex differences in risk factors for future onset of reflux esophagitis
}

\author{
Satoshi Osaga, ${ }^{1}$ Koji Nakada, ${ }^{2}$ Katsuhiko Iwakiri, ${ }^{3}$ Ken Haruma, ${ }^{4}$ Takashi Joh, ${ }^{5}$ and Takeshi Kamiya, ${ }^{1, *}$ \\ 'Department of Medical Innovation, Nagoya City University Graduate School of Medical Sciences, \\ 1 Kawasumi, Mizuho-cho, Mizuho-ku, Nagoya, Aichi 467-8601, Japan \\ 2Department of Laboratory Medicine, The Jikei University School of Medicine, Minato-ku, Tokyo 105-8461, Japan \\ 3Department of Gastroenterology, Nippon Medical School Graduate School of Medicine, Bunkyo-ku, Tokyo 113-8602, Japan \\ ${ }^{4}$ Department of General Internal Medicine 2, Kawasaki Medical School General Medical Center, Okayama, Okayama 700-8505, Japan \\ ${ }^{5}$ Gamagori City Hospital, Gamagori, Aichi 443-8501, Japan
}

(Received 15 December, 2020; Accepted 25 December, 2020; Published online 3 April, 2021)

Reflux esophagitis is known to be more prevalent in males, and previous studies have suggested sex differences in its risk factors. However, little is known about sex differences in the timecourse of risk factors before reflux esophagitis onset. Thus, we conducted a retrospective longitudinal study using health checkup records. From the records of 230,056 individuals obtained from nine institutes in Japan, we selected 1,558 male reflux esophagitis cases, 3,116 male controls, 508 female reflux esophagitis cases, and 1,016 female controls were selected. We compared timecourses of risk factors between the case and control groups and identified abdominal circumference (AC), diastolic blood pressure, alanine transaminase (ALT), and current smoking in males and body mass index (BMI) in females as sex-specific risk factors. We also found that AC and ALT in males and BMI in females were significantly different between the reflux esophagitis case and control groups during the five years before reflux esophagitis onset. Our results suggest that visceral fat-type obesity and fatty liver in males and higher BMI in females are more frequently observed in reflux esophagitis cases several years before reflux esophagitis onset, and that proactive intervention to lifestyle can help prevent reflux esophagitis in both males and females.

Key Words: sex differences, reflux esophagitis, risk factors, longitudinal study, real world evidence

$\mathrm{R}$ eflux esophagitis (RE) is a disease in which reflux of gastric contents into the esophagus causes superficial erosion of the lower esophagus mucosa. The prevalence of RE has increased in recent years in East Asian countries, including Japan. ${ }^{(1)} \mathrm{RE}$ is known to be more prevalent in males, ${ }^{(2)}$ and recent studies have elucidated that there are sex-specific risk factors for RE, such as high abdominal circumference (AC), ${ }^{(3)}$ visceral fat-type metabolic syndrome, ${ }^{(4)}$ hypertension, ${ }^{(5)}$ and hypertriglyceridemia in males as well as risk factors common to both sexes, including obesity, ${ }^{(5-8)}$ advanced age, ${ }^{(3,5,6)}$ current smoking, ${ }^{(6)}$ hiatal hernia, ${ }^{(3,4,6-9)}$ hyperglycemia, ${ }^{(5,9)}$ and absence of atrophic gastritis..$^{(7,8)}$ However, all of these sex-specific risk factors were found in cross-sectional studies, and little is known about whether their time-courses before RE onset are different from those in healthy controls. If the time-courses of risk factors before RE onset differs from those in healthy controls, it would suggest that preventive interventions targeted at improving the risk factors may reduce the risk of developing RE. Thus, we conducted a multicenter longitudinal study to investigate sexspecific factors associated with future RE onset using health checkup records from Japanese institutions.

\section{Materials and Methods}

This was a multicenter, retrospective, longitudinal study using health checkup records from nine institution (Hidaka Hospital, Gunma; Mitsubishi Mihara Hospital, Hiroshima; Junpukai Health Maintenance Center, Okayama; Shimane Environment and Health Public Corporation, Shimane; Meiwa Hospital, Hyogo; Matsue Red Cross Hospital, Shimane; Okazaki City Medical Association Public Health Center, Aichi; Saiseikai Karatsu Hospital, Saga; Shinko Hospital, Hyogo) in Japan. This study was conducted in accordance with the Declaration of Helsinki (7th revision, 2013) and was approved by the ethics committee of each institution and the Central Ethics Committee of the Japanese Association for the Promotion of State-of-the-Art in Medicine, Nagoya, Aichi, Japan.

Population. Nine Japanese institutions that met the following criteria participated in this study: 1) patient information including age, sex, body height, body weight, drinking status, smoking status, fasting blood sugar (FBS), glycated hemoglobin level (HbA1c), and records of upper gastrointestinal endoscopy were available from annual health checkup records and 2) health checkup records from at least four successive years were available. An initial dataset was obtained from individuals who participated in annual health checkups and received at least one upper gastrointestinal endoscopy in 10 years (between April 2004 and March 2014) at the participating institutions. From the individuals who were endoscopically diagnosed with RE, case candidates who met the following criteria were selected: 1) participated in four or more health checkups at the participating institutions between April 2004 and March 2014; 2) newly diagnosed with RE after April 2009; 3) completed an upper gastrointestinal endoscopy during the two years prior to their first $\mathrm{RE}$ diagnosis; 4) participated in three or more health checkups in the five years before their first RE diagnosis; and 5) were 30 or older at their first RE diagnosis. From the individuals without RE diagnosis, control candidates who met the following criteria were selected: 1) participated in four or more health checkups between April 2004 and March 2014 and 2) completed an upper gastrointestinal endoscopy between April 2009 and March 2014. In case-control matching, two control candidates, who were matched in age, sex, participating institution, and underwent upper gastrointestinal endoscopy in the same year as the corresponding case of RE was endoscopically diagnosed, were selected for every one case candidate.

*To whom correspondence should be addressed. E-mail: kamitake@med.nagoya-cu.ac.jp 
Measures. For cases of RE, data obtained at initial RE diagnosis, referred to as the baseline year, and five years before diagnosis were analyzed. For control subjects, data obtained during the same year as the corresponding baseline year of the matched RE case and five years prior were used for analysis. The presence of RE was defined as having grade A or more severe erosion according to the Los Angeles (LA) classification from endoscopic observation or RE being reported in the health checkup records if the LA grade was unavailable. ${ }^{(10)}$ To investigate factors associated with future RE onset, the following clinical parameters were extracted from health checkup records of all subjects: age, sex, body mass index (BMI), FBS, HbAlc, $\mathrm{AC}$, systolic blood pressure (SBP), diastolic blood pressure (DBP), triglyceride (TG), high-density lipoprotein cholesterol (HDL-C), low-density lipoprotein cholesterol (LDL-C), total cholesterol (TC), uric acid (UA), aspartate aminotransferase (AST), alanine aminotransferase (ALT), $\gamma$-glutamyl transpeptidase $(\gamma-\mathrm{GTP})$, drinking status, smoking status, presence or absence of gastrointestinal symptoms (i.e., acid reflux symptoms, gastric pain, heavy stomach, feeling of fullness, and belching), comorbidities (i.e., diabetes, hypertension, and hyperlipidemia), and findings of upper gastrointestinal endoscopy (i.e., hiatal hernia, atrophic gastritis, and Barrett esophagus). Of all the items, gastrointestinal symptoms and comorbidities were investigated by self-administered questionnaires designed by each institute. Hiatal hernia, atrophic gastritis, and Barrett esophagus, whose data were obtained from records of upper gastrointestinal endoscopy of each institution, were endoscopically diagnosed according to the Makuuchi classification, (11) Kimura-Takemoto classification, ${ }^{(12)}$ and Japanese general criteria using palisade vessels as a landmark of esophagogastric junction, ${ }^{(13)}$ respectively in principle.

Statistical analyses. Comparisons at baseline and subsequent time-course analysis of subjects' characteristics were performed separately for the male and female subgroups. For baseline comparisons, we created 100 imputed datasets for missing values in the baseline characteristics using multiple imputation by chained equations and then performed univariate and multivariate logistic regression analyses with the groups as the dependent variable and the baseline characteristics as independent variables. A forward-backward stepwise model selection method based on Akaike's An Information Criterion was applied to determine the characteristics included in the multivariate analysis, and a pooled result of the multivariate logistic regression analyses with differently selected characteristics was estimated by referencing van Buuren's implementation of a simple majority method. ${ }^{(14)}$

In the time-course analysis, we performed regression analyses using a mixed-effect model for repeated measures for each of the continuous characteristics that were significantly associated with the groups in the multivariate analysis for the baseline characteristics. In this model, group, year, and their interaction were entered as fixed effects and subject ID as a random effect, and we assessed the difference in time-course of the characteristics between groups by two aspects: a longitudinal comparison over the whole period using a type III analysis of variance with Satterthwaite's method and a cross-sectional comparison of each year before baseline based on the coefficient of the interaction term. A multivariate logistic regression model with group year, and their interaction was applied as independent variables for categorical characteristics, and the difference in time-course between the groups was assessed via a chi-square test for longitudinal comparison and via a coefficient of the interaction term for cross-sectional comparison.

All statistical tests were performed in a two-sided manner, and the significance level was set at 0.05 . All statistical processing was implemented using R ver. 3.6.3 (R Foundation for Statistical Computing) with lme 4 , lmerTest, emmeans, and mice packages.

\section{Results}

Baseline characteristics. After case-control matching, 1,558 male RE cases, 3,116 male controls, 508 female RE cases, and 1,016 female controls were included in the final analysis (Fig. 1). Baseline characteristics of the male and female RE case and control groups are presented in Table 1. The following characteristics were significantly different between the RE case and control groups in the univariate logistic regression analysis: FBS, SBP, TG, HDL-C, AST, $\gamma$-GTP, current smoking, atrophic gastritis, hypertension, and hyperlipidemia in males and BMI, AC, UA, ALT, acid reflux symptoms, and hiatal hernia in both sexes. The multivariate analysis for the baseline characteristics identified significant risk factors as follows: AC, DBP, ALT, current smoking in males, BMI in females, and acid reflux symptoms, hiatal hernia, and absence of atrophic gastritis in both sexes (Table 2).

Time-course analysis. In the longitudinal comparison of the time-course of risk factors that were significantly associated with $\mathrm{RE}$ in the baseline multivariate analysis, the following factors demonstrated significant differences in changes, during the five years prior to RE diagnosis, between RE cases and healthy controls: AC, ALT, and acid reflux symptoms in males and hiatal hernia in both sexes. The cross-sectional comparisons of the risk factors at each year before baseline revealed significant differences between the groups (RE vs controls) at three or more consecutive years in the following factors: AC, current smoking, hiatal hernia, and atrophic gastritis in males; BMI in females; and acid reflux symptoms in both sexes (Fig. 2 for males, Fig. 3 for females).

\section{Discussion}

In the present study, we conducted a longitudinal analysis to examine sex differences in risk factors for future RE onset using health checkup records from nine institutions in Japan. We identified AC, DBP, ALT, and current smoking in males and BMI in females as sex-specific risk factors. Furthermore, we also found that some of the risk factors had different time-courses between the RE case and control groups before RE onset.

For both male and female subjects, there were significant differences in the measures of obesity between the RE case and control groups in the baseline, longitudinal, and cross-sectional comparisons. However, AC was a risk factor for males, whereas BMI was a risk factor for females. This discrepancy is consistent with findings from two studies by Sogabe et al., ${ }^{(4,9)}$ which demonstrated that visceral fat-dominant metabolic syndrome is a risk factor for RE in males but not in females. Since visceral fat-type obesity is more common in males than in females and a high AC increases esophageal acid exposure, ${ }^{(15,16)}$ it seems reasonable that $\mathrm{AC}$ would be a risk factor for $\mathrm{RE}$ in males specifically. Adiponectin, whose protective effect on mucosal inflammation in esophagus was suggested by Tae et al., ${ }^{(17)}$ was known to inversely correlate with amount of visceral fat, ${ }^{(18)}$ and visceral fat-type obesity in males might cause reflux esophagitis via a decrease of adiponectin level. In addition, the risk of RE in females has a U-shaped relationship with $\mathrm{AC}$, whereby low or high $\mathrm{AC}$ increases the risk of RE compared to medium $\mathrm{AC}$, while in males, it increases monotonically. ${ }^{(19)}$ This may indicate why $\mathrm{AC}$ was not identified as a risk factor for $\mathrm{RE}$ in females.

The present study identified DBP, ALT, and current smoking as male-specific risk factors for RE. First, elevated ALT in male $\mathrm{RE}$ patients is believed to indicate the presence of a liver disease, which is consistent with the study of Yang et al., ${ }^{(20)}$ which claimed that nonalcoholic fatty liver disease is a risk factor for RE. Second, current smoking was a significant risk factor for $\mathrm{RE}$ in males, similar to previous studies. ${ }^{(6,8)}$ On the other hand, our results demonstrate that current smoking is not a risk factor 


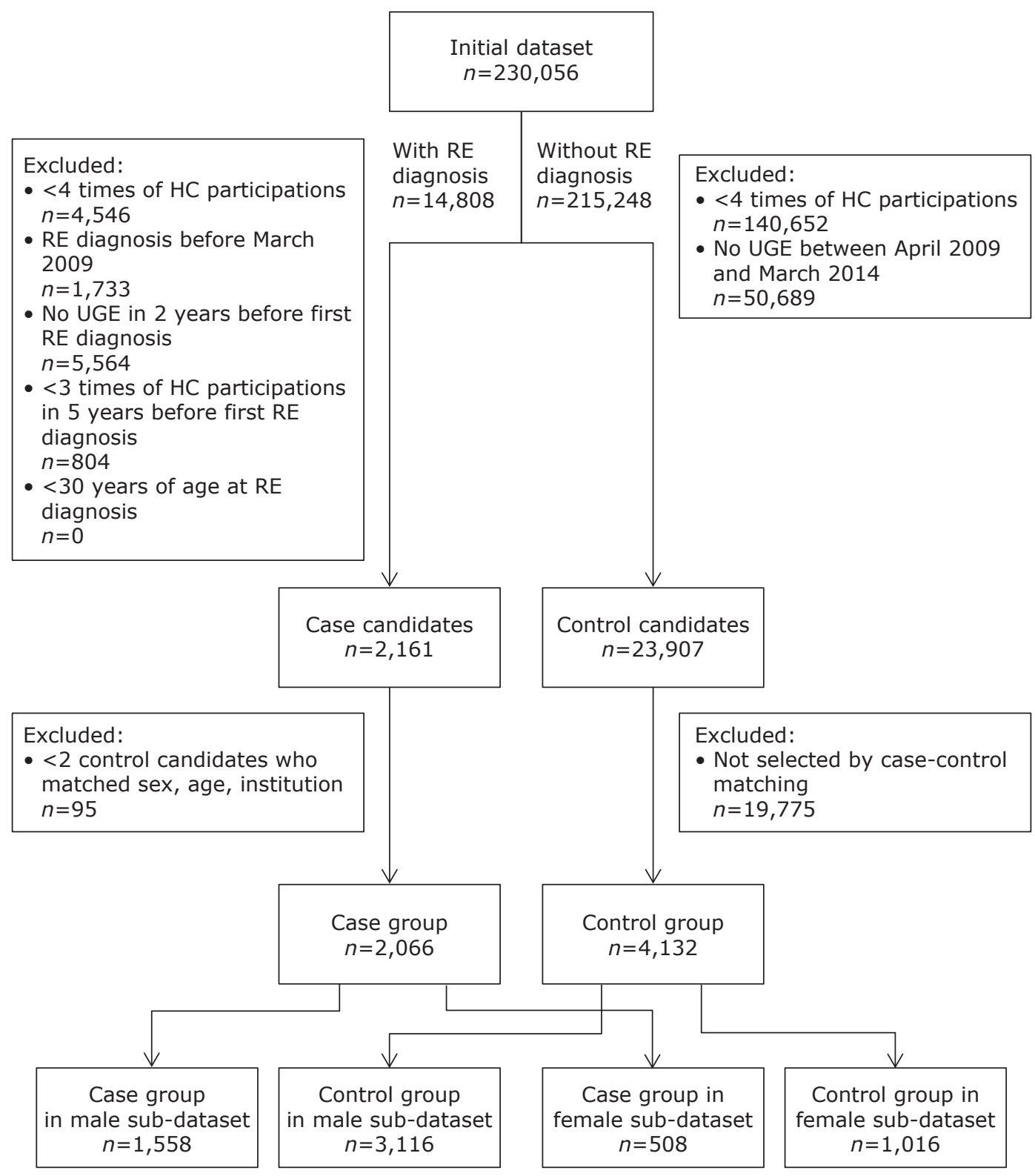

Fig. 1. Subject flow in this study. RE, reflux esophagitis; HC, health checkup; UGE, upper gastrointestinal endoscopy.

for RE in females, which is similar to one of the studies and differs from the other. ${ }^{(6,8)}$ It is likely that insufficient statistical power due to the low rate of smoking among females led to these controversial results, and a larger study is needed to investigate sex differences in smoking as a risk factor for RE. Finally, elevated DBP in males was also a significant risk factor for RE in the baseline multivariate analysis, which is partially consistent with the study by Moki et al., ${ }^{(5)}$ wherein hypertension, defined as SBP $\geq 140 \mathrm{mmHg}$ and/or DBP $\geq 90 \mathrm{mmHg}$, was shown to be a risk factor for RE in males. However, considering that there were no significant differences between the groups in the time-course of DBP, the impact of DBP, even if it was associated with RE, may be minor.

The presence of hiatal hernia and the absence of atrophic gastritis have been reported as risk factors for RE in many studies and were significantly associated with RE onset in the baseline comparisons in this study. ${ }^{(3,4,6-9)}$ The longitudinal comparisons of the prevalence of hiatal hernia between groups demonstrated significantly more hiatal hernias in the RE case group than in the control group in both males and females. Additionally, the prevalence of hiatal hernia was significantly higher in the RE case group than in the control group during the three years prior to RE onset in males, and one year prior to RE onset in females. Hiatal hernia is considered to be one of the major etiologies of $\mathrm{RE}^{(21)}$ and the fact that the prevalence of hiatal hernia increased before RE onset confirms this assertion. There was no significant difference in the prevalence of atrophic gastritis in the time-course of RE between the RE case and control groups in both sexes in the longitudinal comparisons. Furthermore, the rate of atrophic gastritis was lower in the RE case group for five consecutive years prior to RE onset. This is a reasonable result as gastric mucosal atrophy, mainly caused by Helicobacter pylori, is a long-term process of deterioration over a period of years to decades and typically provokes suppression of acid secretion. ${ }^{(22)}$ In contrast, the differences in atrophic gastritis between groups were unclear in both the longitudinal 
Table 1. Baseline characteristics of the case and control groups by sex

\begin{tabular}{|c|c|c|c|c|c|c|}
\hline & & Male & & & Female & \\
\hline & $\begin{array}{c}\text { Case } \\
(n=1,558)\end{array}$ & $\begin{array}{c}\text { Control } \\
(n=3,116)\end{array}$ & $p$ value $^{\dagger}$ & $\begin{array}{c}\text { Case } \\
(n=508)\end{array}$ & $\begin{array}{c}\text { Control } \\
(n=1,016)\end{array}$ & $p$ value $^{\dagger}$ \\
\hline Age $(y)$, Mean \pm SD & $53.9 \pm 9.6$ & $54.0 \pm 9.7$ & 0.805 & $54.1 \pm 8.4$ & $53.9 \pm 8.3$ & 0.692 \\
\hline BMI $\left(\mathrm{kg} / \mathrm{m}^{2}\right)$, Mean \pm SD & $23.9 \pm 3.1$ & $23.3 \pm 2.9$ & $<0.001$ & $22.2 \pm 3.4$ & $21.6 \pm 3.1$ & 0.003 \\
\hline$A C(\mathrm{~cm})$, Mean $\pm S D$ & $85.7 \pm 8.4$ & $83.8 \pm 8.0$ & $<0.001$ & $79.7 \pm 9.4$ & $78.5 \pm 8.8$ & 0.016 \\
\hline $\mathrm{FBS}(\mathrm{mg} / \mathrm{dl})$, Mean $\pm \mathrm{SD}$ & $104.6 \pm 18.8$ & $102.9 \pm 17.6$ & 0.002 & $96.8 \pm 14.7$ & $96.7 \pm 13.4$ & 0.807 \\
\hline $\operatorname{HbA} 1 \mathrm{c}(\%)$, Mean $\pm \mathrm{SD}$ & $5.71 \pm 0.61$ & $5.68 \pm 0.57$ & 0.098 & $5.63 \pm 0.45$ & $5.63 \pm 0.44$ & 0.935 \\
\hline $\mathrm{SBP}(\mathrm{mmHg})$, Mean $\pm \mathrm{SD}$ & $113.2 \pm 22.9$ & $111.4 \pm 22.5$ & 0.011 & $110.4 \pm 20.1$ & $109.7 \pm 20.0$ & 0.491 \\
\hline $\mathrm{DBP}(\mathrm{mmHg})$, Mean \pm SD & $86.7 \pm 22.0$ & $85.5 \pm 22.1$ & 0.069 & $76.6 \pm 20.2$ & $75.8 \pm 19.5$ & 0.456 \\
\hline $\mathrm{TG}(\mathrm{mg} / \mathrm{dl})$, Mean $\pm \mathrm{SD}$ & $137.7 \pm 93.0$ & $124.7 \pm 77.5$ & $<0.001$ & $92.0 \pm 68.0$ & $87.4 \pm 46.9$ & 0.127 \\
\hline $\mathrm{HDL}-\mathrm{C}(\mathrm{mg} / \mathrm{dl})$, Mean $\pm \mathrm{SD}$ & $57.4 \pm 14.7$ & $58.5 \pm 14.5$ & 0.012 & $69.7 \pm 15.8$ & $70.8 \pm 16.0$ & 0.2 \\
\hline LDL-C (mg/dl), Mean \pm SD & $122.0 \pm 30.0$ & $121.8 \pm 30.0$ & 0.808 & $119.4 \pm 29.7$ & $120.8 \pm 29.9$ & 0.399 \\
\hline $\mathrm{TC}(\mathrm{mg} / \mathrm{dl})$, Mean $\pm \mathrm{SD}$ & $205.2 \pm 32.1$ & $204.9 \pm 32.5$ & 0.75 & $214.4 \pm 34.6$ & $215.6 \pm 34.0$ & 0.515 \\
\hline $\mathrm{UA}(\mathrm{mg} / \mathrm{dl})$, Mean $\pm \mathrm{SD}$ & $6.08 \pm 1.18$ & $5.97 \pm 1.17$ & 0.004 & $4.54 \pm 1.02$ & $4.42 \pm 0.93$ & 0.027 \\
\hline AST (IU/L), Mean \pm SD & $24.9 \pm 11.6$ & $23.7 \pm 9.1$ & $<0.001$ & $21.7 \pm 8.3$ & $20.9 \pm 6.7$ & 0.059 \\
\hline ALT (IU/L), Mean $\pm S D$ & $28.3 \pm 19.1$ & $25.2 \pm 15.5$ & $<0.001$ & $19.4 \pm 12.3$ & $18.1 \pm 10.0$ & 0.029 \\
\hline$\gamma$-GTP $($ IU/L), Mean \pm SD & $52.7 \pm 53.5$ & $45.7 \pm 47.9$ & $<0.001$ & $24.9 \pm 22.3$ & $22.9 \pm 20.4$ & 0.082 \\
\hline Current drinking, $n(\%)$ & $1,237(79.4)$ & $2,421(77.7)$ & 0.202 & $244(48.0)$ & $463(45.6)$ & 0.378 \\
\hline Current smoking, $n(\%)$ & 466 (29.9) & $826(26.5)$ & 0.018 & $44(8.6)$ & $64(6.3)$ & 0.125 \\
\hline Gastrointestinal symptoms, & & & & & & \\
\hline Acid reflux symptoms & $169(10.8)$ & $131(4.2)$ & $<0.001$ & $72(14.1)$ & $62(6.1)$ & $<0.001$ \\
\hline Gastric pain & $111(7.1)$ & $235(7.5)$ & 0.712 & $84(16.6)$ & $148(14.5)$ & 0.329 \\
\hline Heavy stomach & $267(17.1)$ & $510(16.4)$ & 0.674 & $100(19.7)$ & $154(15.2)$ & 0.057 \\
\hline Feeling of fullness & $203(13.0)$ & $387(12.4)$ & 0.706 & $89(17.5)$ & $131(12.9)$ & 0.057 \\
\hline Belching & $240(15.4)$ & $428(13.7)$ & 0.329 & $76(14.9)$ & $140(13.8)$ & 0.615 \\
\hline Endoscopic findings, $n(\%)$ & & & & & & \\
\hline Hiatal hernia & $388(24.9)$ & $453(14.5)$ & $<0.001$ & $95(18.7)$ & $64(6.3)$ & $<0.001$ \\
\hline Atrophic gastritis & $572(36.7)$ & $1377(44.2)$ & $<0.001$ & $155(30.5)$ & 339 (33.4) & 0.282 \\
\hline Barrett's esophagus & $44(2.8)$ & 109 (3.5) & 0.22 & $13(2.6)$ & $29(2.8)$ & 0.867 \\
\hline Comorbidities, $n(\%)$ & & & & & & \\
\hline Diabetes & $153(9.8)$ & $258(8.3)$ & 0.083 & $27(5.3)$ & $47(4.6)$ & 0.554 \\
\hline Hypertension & $366(23.5)$ & $619(19.9)$ & 0.005 & $68(13.3)$ & $115(11.3)$ & 0.263 \\
\hline Hyperlipidemia & $282(18.1)$ & $492(15.8)$ & 0.048 & $83(16.3)$ & $154(15.1)$ & 0.571 \\
\hline
\end{tabular}

Missing values were imputed by chained equations. ${ }^{\dagger} p$ values were calculated based on pooled estimates of univariate logistic regression coefficients with the group as dependent variable. $n$, total number of subjects in the group (except subjects whose characteristics were unknown); BMI, body mass index; AC, abdominal circumference; FBS, fasting blood sugar; HbA1c, glycated hemoglobin levels; SBP, systolic blood pressure; DBP, diastolic blood pressure; TG, triglyceride; HDL-C, high-density lipoprotein cholesterol; LDL-C, low-density lipoprotein cholesterol; TC, total cholesterol; UA, uric acid; AST, aspartate aminotransferase; ALT, alanine aminotransferase; $\gamma$-GTP, $\gamma$-glutamyl transpeptidase.

Table 2. Result of multivariate logistic regression analysis for baseline characteristics (only selected variables by stepwise method)

\begin{tabular}{|c|c|c|c|c|}
\hline & \multicolumn{2}{|l|}{ Male } & \multicolumn{2}{|l|}{ Female } \\
\hline & Odds ratio $[95 \% \mathrm{Cl}]$ & $p$ value & Odds ratio $[95 \% \mathrm{Cl}]$ & $p$ value \\
\hline BMI $\left(\mathrm{kg} / \mathrm{m}^{2}\right)$ & - & - & $1.037[1.001,1.074]$ & 0.046 \\
\hline $\mathrm{AC}^{\dagger}(\mathrm{cm})$ & $1.022[1.014,1.031]$ & $<0.001$ & - & - \\
\hline $\mathrm{DBP}^{\dagger}(\mathrm{mmHg})$ & $1.003[1.00003,1.006]$ & 0.048 & - & - \\
\hline $\mathrm{UA}^{\dagger}(\mathrm{mg} / \mathrm{dl})$ & - & - & $1.102[0.973,1.249]$ & 0.127 \\
\hline $\mathrm{ALT}^{\dagger}$ (IU/L) & $1.005[1.001,1.009]$ & 0.009 & - & - \\
\hline Current smoking & $1.197[1.037,1.382]$ & 0.014 & - & - \\
\hline Acid reflux symptoms & $2.703[2.117,3.450]$ & $<0.001$ & $2.548[1.760,3.687]$ & $<0.001$ \\
\hline Hiatal hernia & $2.189[1.862,2.574]$ & $<0.001$ & $3.598[2.468,5.245]$ & $<0.001$ \\
\hline Atrophic gastritis & $0.646[0.565,0.738]$ & $<0.001$ & $0.713[0.552,0.920]$ & 0.009 \\
\hline
\end{tabular}

blood pressure; ALT, alanine aminotransferase. 
A

(cm)

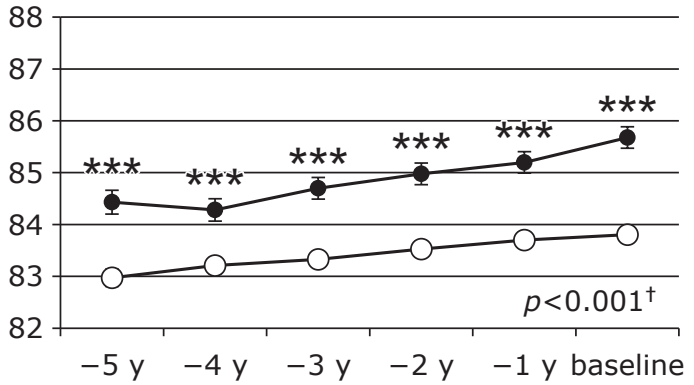

C

(IU/L)

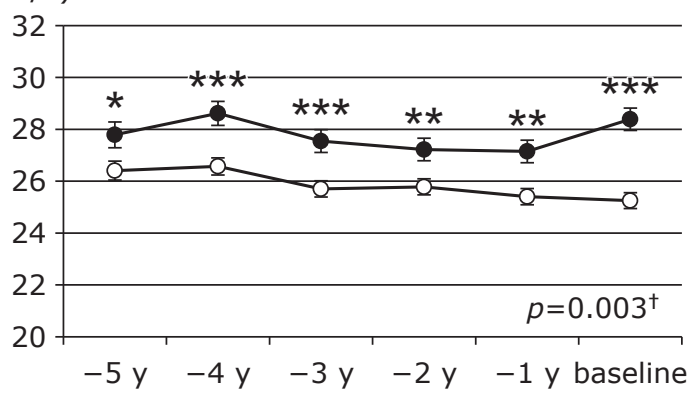

E

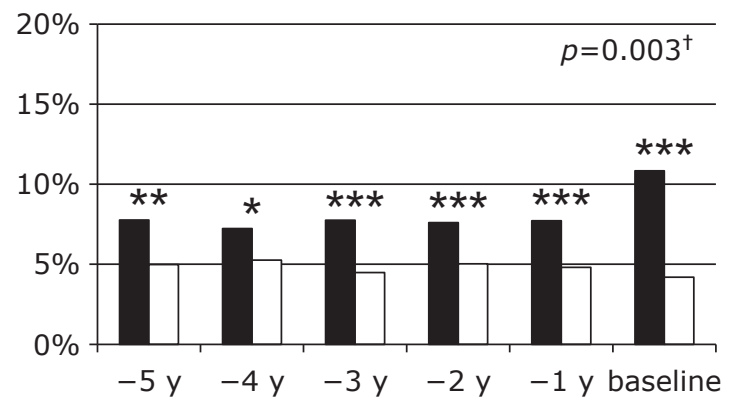

B

$(\mathrm{mmHg})$

Diastolic blood pressure

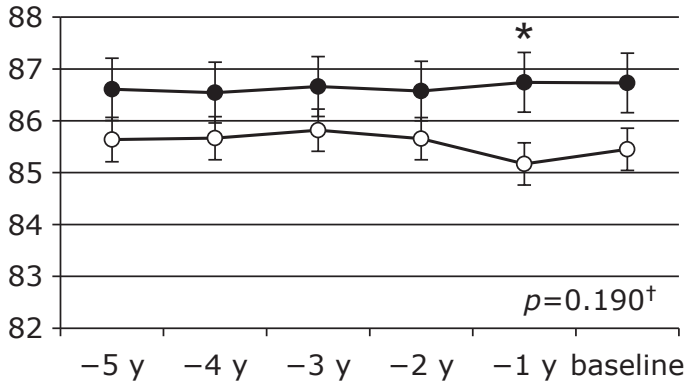

D

\section{Current smoking}

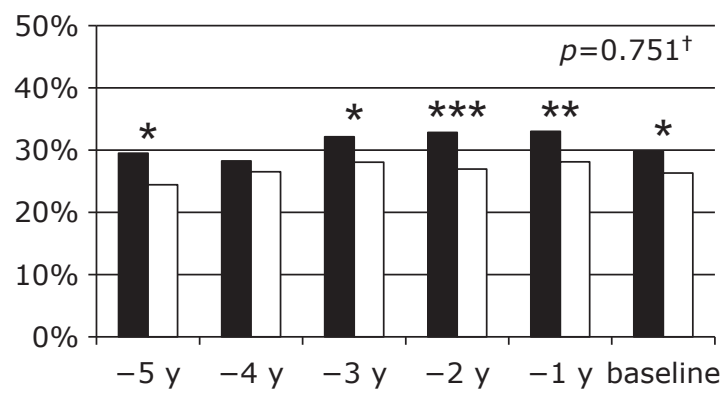

$\mathrm{F}$

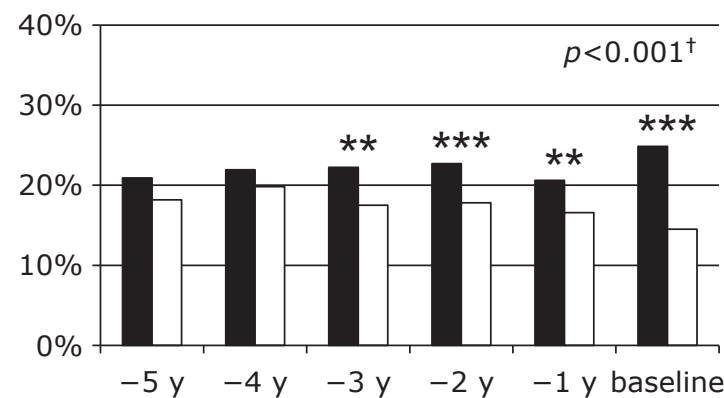

G

Atrophic gastritis

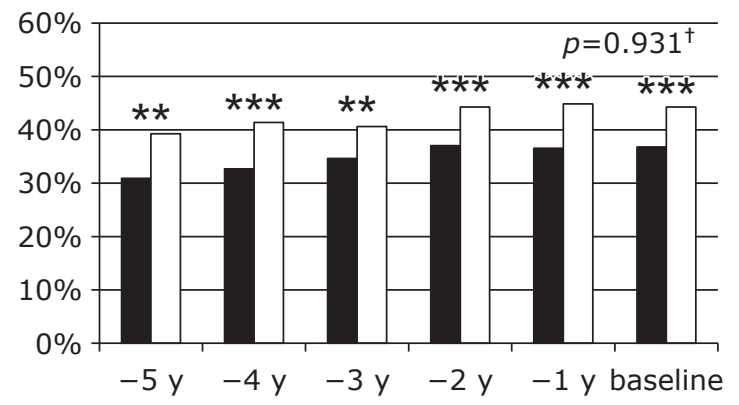

- - Case group

$-\circ$ Control group

Fig. 2. Result of time-course analysis of factors in males. For continuous variables, points and error bars denote least square means at each year and its SE, respectively. For categorical variables, boxes denote percentages at each year. ${ }^{*} p<0.05 ;{ }^{* *} p<0.01 ; * * * p<0.001$ in the cross-sectional comparisons between the groups. ${ }^{\dagger} p$ value in the longitudinal comparison between the groups.

and cross-sectional comparisons in females. Although there is no rational explanation for this sex difference, postmenopausal progression of atrophy may have confounded the association between atrophic gastritis and RE in females. ${ }^{(23)}$
Since the prevalence of reflux symptoms was significantly higher in the RE case group than in the control group for the five years prior to RE onset, the presence of the symptoms is an obvious risk factor of RE for both males and females. 
A

$\left(\mathrm{kg} / \mathrm{m}^{2}\right)$

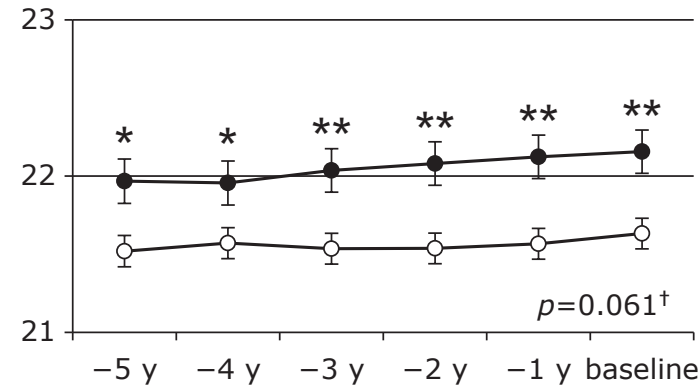

C

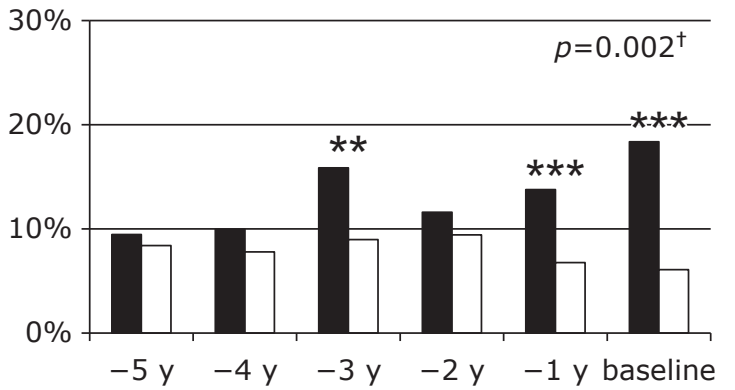

B

Acid reflux symptoms

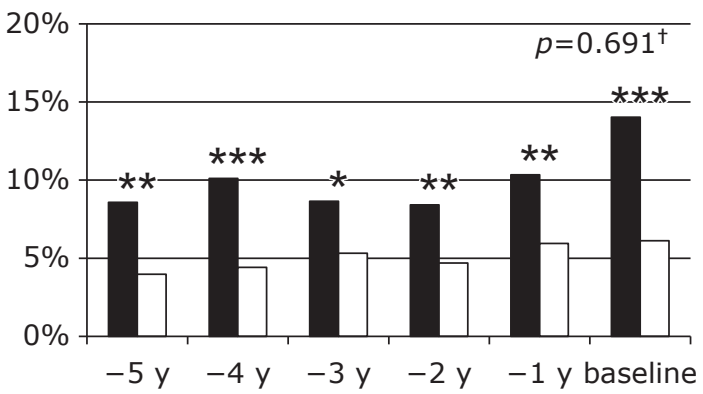

D

Atrophic gastritis

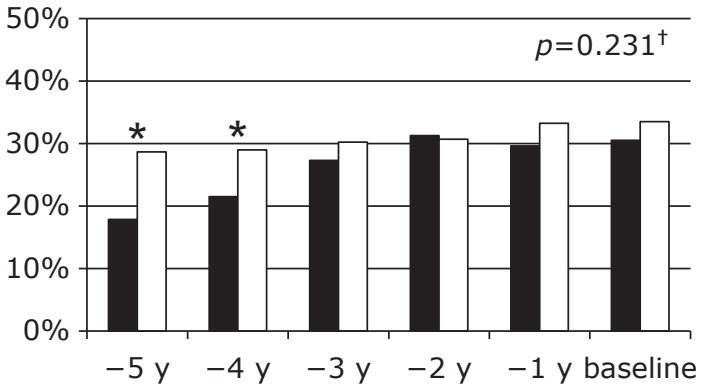

\section{- Case group}

- $\square$ Control group

Fig. 3. Result of time-course analysis of factors in females. For continuous variables, points and error bars denote least square means at each year and its $\mathrm{SE}$, respectively. For categorical variables, boxes denote percentages at each year. ${ }^{*} p<0.05 ;{ }^{* *} p<0.01 ;{ }^{* * *} p<0.001$ in the cross-sectional comparisons between the groups. ${ }^{\dagger} p$ value in the longitudinal comparison between the groups.

This is not surprising, given that RE is a disease primarily caused by acid reflux, ${ }^{(21)}$ but it is noteworthy that acid reflux symptoms were more prevalent for several years before RE onset in the RE case group. However, it should be noted that the prevalence of acid reflux symptoms was likely skewed toward lower values. In our cohort, the asymptomatic rate at the RE onset was $90.2 \%$ in males and $86.0 \%$ in females. This is much higher than the $33.6-41.7 \%$ reported in a systematic review. ${ }^{(24)}$ Although medication records were not available from the health checkup record database, it is speculated that this may be because some subjects were being treated for reflux symptoms.

This study has several limitations. We had planned to collect data regarding history of medications that could affect RE development, namely acid secretion inhibitors, calcium blockers, and aspirin, at first; however we abandoned because they could not be sufficiently obtained from the health checkup records. In addition, the items directly related to lifestyle habits, such as dietary and exercise habits, could not be also used for analysis because the data format was not standardized among participating institutions. Lack of information about treatment history and inconsistency in data format are major barriers to retrospective studies, in general; therefore we hope that institutions across the country will adopt a standardized format to investigate health information including treatment history and lifestyle habits for health checkup records and revitalize researches based on "real world data" in the future. Moreover, since we defined the presence or absence of RE solely on health checkup records from April 2004 to March 2013 in this study, subjects who had a history of RE before April 2004 or who had been diagnosed with RE and treated outside of their health checkup might not have been included in our cohort. Although this may have caused some bias, it is probably limited as the risk factors associated with RE in this study were consistent with previous studies.

In conclusion, the results of this study demonstrate that acid reflux symptoms, hiatal hernia, and the absence of atrophic gastritis are common risk factors for RE in both males and females, whereas AC, DBP, ALT, and current smoking are specific risk factors to males and BMI is specific to females. Moreover, significant differences between the groups in $\mathrm{AC}$, ALT, acid reflux symptoms, and atrophic gastritis in males and BMI and acid reflux symptoms in females were observed during the five years prior to RE onset. Although the risk factors identified in this study are already known from the previous cross-sectional studies, this is the first study to investigate the time-courses of the factors by year during five years prior to RE onset, presenting important findings to elucidate a natural history of developing RE. In addition, these results suggest that males with visceral fat-type obesity or fatty liver and females with higher BMI are at risk for future RE onset, and that proactive intervention to their lifestyle, including dietary and exercise habit changes can help prevent RE in both males and females.

\section{Author Contributions}

$\mathrm{SO}, \mathrm{KN}, \mathrm{KI}, \mathrm{KH}$, and TJ contributed to the study conception and design. Material preparation, data collection, and analysis were performed by SO. The first draft of the manuscript was written by SO and all the other authors critically commented on previous versions of the manuscript. All authors read and approved the final manuscript. 


\section{Acknowledgments}

This work was funded by AstraZeneca K.K. (Osaka, Japan), based on a contract of clinical study support. We are grateful to Yuzuru Toki (Hidaka Hospital, Gunma, Japan), Ryo Yamauchi (Mitsubishi Mihara Hospital, Hiroshima, Japan), Eizo Kayashima (Junpukai Health Maintenance Center, Okayama, Japan), Kyoichi Adachi (Shimane Environment and Health Public Corporation, Shimane, Japan), Kiyohiko Kishi (Meiwa Hospital, Hyogo, Japan), Hiroshi Suetsugu (Matsue Red Cross Hospital, Shimane, Japan), Tsuneya Wada (Okazaki City Medical Association Public Health Center, Aichi, Japan), Hiroyoshi Endo (Saiseikai Karatsu Hospital, Saga, Japan), and Hajime Yamada (Shinko Hospital, Hyogo, Japan) for providing the health checkup records to this study.

\section{Abbreviations}

$\mathrm{AC}$

abdominal circumference
LA

LDL-C

RE

SBP

TC

TG

UA

alanine aminotransferase aspartate aminotransferase body mass index diastolic blood pressure fasting blood sugar $\gamma$-glutamyl transpeptidase glycated hemoglobin level high-density lipoprotein cholesterol Los Angeles low-density lipoprotein cholesterol reflux esophagitis systolic blood pressure total cholesterol triglyceride uric acid

\section{Conflict of Interest}

No potential conflicts of interest were disclosed.

Missing Data (2nd ed.) Chapman \& Hall/CRC Interdisciplinary Statistics Series. 2018. https://stefvanbuuren.name/fimd/sec-stepwise.html. Accessed 7 Jul 2020.

15 Eguchi M, Tsuchihashi K, Saitoh S, et al. Visceral obesity in Japanese patients with metabolic syndrome: reappraisal of diagnostic criteria by CT scan. Hypertens Res 2007; 30: 315-323.

16 El-Serag HB, Ergun GA, Pandolfino J, Fitzgerald S, Tran T, Kramer JR. Obesity increases oesophageal acid exposure. Gut 2007; 56: 749-755.

17 Tae CH, Jung HK, Kim SE, Jung SA, Jee SH. Potential involvement of adiponectin in obesity-associated erosive esophagitis. $J$ Clin Biochem Nutr 2020; 67: 206-213.

18 Kishida K, Kim KK, Funahashi T, Matsuzawa Y, Kang HC, Shimomura I. Relationships between circulating adiponectin levels and fat distribution in obese subjects. J Atheroscler Thromb 2011; 18: 592-595.

19 Yang HJ, Chang Y, Park SK, et al. Sex differences in the relation between waist circumference within the normal range and development of reflux esophagitis. J Clin Med 2019; 8: 67.

20 Yang HJ, Chang Y, Park SK, et al. Nonalcoholic fatty liver disease is associated with increased risk of reflux esophagitis. Dig Dis Sci 2017; 62: 3605-3613.

21 Dodds WJ, Hogan WJ, Helm JF, Dent J. Pathogenesis of reflux esophagitis. Gastroenterology 1981; 81: 376-394.

22 Sipponen P, Kekki M, Seppälä K, Siurala M. The relationships between chronic gastritis and gastric acid secretion. Aliment Pharmacol Ther 1996; 10 Suppl 1: 103-118.

23 Kato S, Matsukura N, Togashi A, et al. Sex differences in mucosal response to Helicobacter pylori infection in the stomach and variations in interleukin-8, COX-2 and trefoil factor family 1 gene expression. Aliment Pharmacol Ther 2004; 20 Suppl 1: 17-24.

24 Jung HK. Epidemiology of gastroesophageal reflux disease in Asia: a systematic review. J Neurogastroenterol Motil 2011; 17: 14-27. 1557-1566. (in Japanese)

12 Kimura K, Satoh K, Ido K, Taniguchi Y, Takimoto T, Takamoto T. Gastritis in the Japanese stomach. Scand J Gastroenterol Suppl 1996; 214: 17-23.

13 Ishimura N, Amano Y, Kinoshita Y. Endoscopic definition of esophagogastric junction for Barrett's esophagus: importance of systematic education and training. Dig Endosc 2009; 21: 213-218.

14 van Buuren S. 5.4 Stepwise model selection. In: Flexible Imputation of
This is an open access article distributed under the terms of the Creative Commons Attribution-NonCommercial-NoDerivatives License (http://creativecommons.org/licenses/by-nc-nd/4.0/). 